\title{
Index60 as an additional diagnostic criterion for type 1 diabetes
}

\author{
Maria J. Redondo ${ }^{1}$ (D) - Brandon M. Nathan ${ }^{2}$ - Laura M. Jacobsen ${ }^{3}$ - Emily Sims ${ }^{4}$ - Laura E. Bocchino ${ }^{5,6}$. \\ Alberto Pugliese ${ }^{7}$. Desmond A. Schatz ${ }^{3}$. Mark A. Atkinson ${ }^{3}$. Jay Skyler ${ }^{7}$. Jerry Palmer ${ }^{8}$. Susan Geyer ${ }^{5,9}$. \\ Jay M. Sosenko ${ }^{7}$. for the Type 1 diabetes TrialNet Study Group
}

Received: 22 May 2020 / Accepted: 28 October 2020 / Published online: 26 January 2021

(C) The Author(s), under exclusive licence to Springer-Verlag GmbH, DE part of Springer Nature 2021

\begin{abstract}
Aims/hypothesis We aimed to compare characteristics of individuals identified in the peri-diagnostic range by Index60 (composite glucose and C-peptide measure) $\geq 2.00,2 \mathrm{~h}$ OGTT glucose $\geq 11.1 \mathrm{mmol} / 1$, or both.

Methods We studied autoantibody-positive participants in the Type 1 Diabetes TrialNet Pathway to Prevention study who, at their baseline OGTT, had $2 \mathrm{~h}$ blood glucose $\geq 11.1 \mathrm{mmol} / 1 \mathrm{and} /$ or Index60 $\geq 2.00(n=354$, median age $=11.2$ years, age range $=1.7-$ 46.6; $49 \%$ male, $83 \%$ non-Hispanic White). Type 1 diabetes-relevant characteristics (e.g., age, C-peptide, autoantibodies, BMI) were compared among three mutually exclusive groups: $2 \mathrm{~h}$ glucose $\geq 11.1 \mathrm{mmol} / \mathrm{l}$ and Index $60<2.00[\mathrm{Glu}(+), n=76], 2 \mathrm{~h}$ glucose $<11.1 \mathrm{mmol} / 1$ and Index $60 \geq 2.00[\operatorname{Ind}(+), n=113]$, or both $2 \mathrm{~h}$ glucose $\geq 11.1 \mathrm{mmol} / 1$ and $\operatorname{Index} 60 \geq 2.00[\mathrm{Glu}(+) / \operatorname{Ind}(+), n=165]$. Results Participants in Glu(+), vs those in Ind(+) or Glu(+)/Ind(+), were older (mean ages $=22.9$, 11.8 and 14.7 years, respectively), had higher early (30-0 min) C-peptide response (1.0, 0.50 and $0.43 \mathrm{nmol} / \mathrm{l})$, higher AUC C-peptide (2.33, 1.13 and $1.10 \mathrm{nmol} / \mathrm{l})$, higher percentage of overweight/obesity $(58 \%, 16 \%$ and $30 \%)$ (all comparisons, $p<0.0001)$, and a lower percentage of multiple autoantibody positivity $(72 \%, 92 \%$ and 93\%) $(p<0.001)$. OGTT-stimulated C-peptide and glucose patterns of Glu(+) differed appreciably from Ind(+) and Glu(+)/Ind(+). Progression to diabetes occurred in 61\% (46/76) of Glu(+) and 63\% $(71 / 113)$ of Ind(+). Even though Index60 $\geq 2.00$ was not a Pathway to Prevention diagnostic criterion, Ind(+) had a 4 year cumulative diabetes incidence of $95 \%$ (95\% CI 86\%, 98\%).

Conclusions/interpretation Participants in the Ind $(+)$ group had more typical characteristics of type 1 diabetes than participants in the $\mathrm{Glu}(+)$ did and were as likely to be diagnosed. However, unlike Glu(+) participants, Ind(+) participants were not identified at the baseline OGTT.
\end{abstract}

Keywords C-peptide - Diagnosis · Glucose $\cdot$ Heterogeneity · Index60 - Insulin resistance $\cdot$ Prediction $\cdot$ TrialNet $\cdot$ Type 1 diabetes · Type 2 diabetes

Abbreviations

GCRC Glucose and C-peptide response curve
$\operatorname{Glu}(+)$

Index $60<2.00$
Complete listing of Type 1 Diabetes TrialNet Study Group members is included in the electronic supplementary material (ESM)

Maria J. Redondo

redondo@bcm.edu

1 Texas Children's Hospital, Baylor College of Medicine, Houston, TX, USA

2 Division of Pediatric Endocrinology, University of Minnesota, Minneapolis, MN, USA

3 University of Florida Diabetes Institute, Gainesville, FL, USA

4 Indiana University School of Medicine, Indiana University, Indianapolis, IN, USA
University of South Florida, Tampa, FL, USA

6 Jaeb Center for Health Research, Tampa, FL, USA

7 Diabetes Research Institute, Miller School of Medicine, University of Miami, Miami, FL, USA

8 University of Washington, Seattle, WA, USA

9 Division of Biomedical Statistics and Informatics, Mayo Clinic, Rochester, MN, USA 


\section{Research in context}

\section{What is already known about this subject?}

- In islet autoantibody-positive individuals, glucose criteria alone are currently used to diagnose type 1 diabetes

- Beta cell function deficiency is a key characteristic of type 1 diabetes that is currently not used to diagnose type 1 diabetes

- $\quad$ Because type 2 diabetes is prevalent in the population, some autoantibody-positive individuals could develop type 2 diabetes, but the current diagnostic strategy would classify their diabetes as type 1

\section{What is the key question?}

- Do individuals with Index60 values $\geq 2.00$ and $2 \mathrm{~h}$ glucose levels $<11.1 \mathrm{mmol} / \mathrm{l}$ have more typical characteristics of type 1 diabetes and are they as likely to be diagnosed as individuals who have $2 \mathrm{~h}$ glucose levels $\geq 11.1 \mathrm{mmol} / \mathrm{l}$ and Index60 values $<2.00$ ?

What are the new findings?

- Among islet autoantibody-positive individuals, an Index60 value $\geq 2.00$ identifies more individuals with characteristics typical of type 1 diabetes than glucose criteria alone

- Index60 values $\geq 2.00$ can be used to diagnose metabolic abnormalities characteristic of type 1 diabetes earlier than glucose criteria alone

How might this impact on clinical practice in the foreseeable future?

- Index60 values $\geq 2.00$ can be used in addition to glucose criteria to provide an earlier and more accurate diagnosis of type 1 diabetes among autoantibody-positive individuals

$\begin{array}{ll}\text { Glu(+)/Ind(+) } & \begin{array}{l}2 \mathrm{~h} \text { glucose } \geq 11.1 \mathrm{mmol} / \mathrm{l} \text { and } \\ \text { Index } 60 \geq 2.00\end{array} \\ \text { IA-2A } & \begin{array}{l}\text { Insulinoma-associated } \\ \text { antigen-2 autoantibody } \\ \text { Islet cell antibodies }\end{array} \\ \text { ICA } & 2 \mathrm{~h} \text { glucose }<11.1 \mathrm{mmol} / \mathrm{l} \text { and } \\ \text { Ind(+) } & \text { Index } 60 \geq 2.00 \\ \text { mIAA } & \text { Microinsulin autoantibody assay } \\ \text { PTP } & \text { Pathway to Prevention } \\ \text { ZnT8A } & \text { Zinc transporter eight autoantibodies }\end{array}$

\section{Introduction}

Standard glucose criteria for both type 1 and type 2 diabetes [1] are generally used to diagnose type 1 diabetes in autoantibody-positive participants in longitudinal research studies. Specifically, among asymptomatic individuals, a $2 \mathrm{~h}$ blood glucose $\geq 11.1 \mathrm{mmol} / 1$ during an OGTT, confirmed in a second test, is the diagnostic criterion most often utilised in prevention trials. However, this standard approach does not assess decreased insulin secretion, a major factor in the development of type 1 diabetes.

Individuals with positive islet autoantibodies who ultimately exceed the glucose criteria mentioned above, either clinically or in research studies, do not necessarily have classic type 1 diabetes, as observed in typically young, thin individuals who have type 1 diabetes-associated genetic factors, develop multiple islet autoantibodies and lose their ability to make insulin relatively quickly [1-6]. These individuals could have developed an atypical form of type 1 diabetes. The heterogeneity of type 1 diabetes is a recognised barrier for the success of clinical trials aimed at halting the development and progression of type 1 diabetes [7]. Moreover, since type 2 diabetes is so prevalent, it is likely that a certain percentage of individuals diagnosed as having type 1 diabetes may actually have type 2 diabetes. This lack of diagnostic clarity could potentially be improved by additional metabolic criteria that also take insulin secretion into account, such as those that include both glucose and C-peptide.

This potential diagnostic application was evident in a study of Index60 [8], a composite measure of fasting C-peptide, 60 min C-peptide and 60 min glucose derived from a proportional hazards model. Index60 was initially developed in autoantibody-positive individuals in the Diabetes Prevention Trial (DPT)-1 trial [8] as an additional metabolic diagnostic criterion to facilitate an earlier diagnosis of type 1 diabetes. The findings showed that Index 60 values $\geq 2.00$ were highly predictive of type 1 diabetes approximately 6-12 months earlier than standard glucose measures alone were. This corroborated findings of Index60 values $\geq 2.00$ reported in the TrialNet Pathway to Prevention (PTP) cohort. In a separate TrialNet PTP cohort, we assessed the utility of an Index60 threshold $\geq 1.00$ as a prediabetic endpoint in autoantibodypositive individuals, and found that Index 60 could better identify individuals with typical characteristics of type 1 diabetes 
than dysglycaemia (of which the $2 \mathrm{~h}$ glucose is the main component) [9]. Participants above the 1.00 Index60 threshold were appreciably younger and had greater frequencies of islet autoantibodies than those who had dysglycaemia. Not surprisingly, individuals above the 1.00 threshold also had markedly lower C-peptide levels.

Here, we aimed to determine whether Index60, as a diagnostic endpoint, could identify individuals with typical characteristics of type 1 diabetes, who would not have been detected during early stages of disease progression by glucose criteria alone. Demographic, immunological, genetic and metabolic characteristics were analysed among 354 non-diabetic, autoantibody-positive participants from the TrialNet PTP study $[10,11]$ who exceeded $2 \mathrm{~h}$ glucose values $\geq 11.1 \mathrm{mmol} / 1$ and/or Index $60 \geq 2.00$ thresholds at their baseline OGTT. Individuals included in this analysis were mutually exclusive of those in the prior study pertaining to diagnosis [8], since all included in that study had baseline $2 \mathrm{~h}$ glucose values $<11.1 \mathrm{mmol} / \mathrm{l}$ and Index60 values $<2.00$. Using this unique cohort, we performed the first systematic study to assess the use of a composite measure of glucose and C-peptide, such as Index60, for diagnosing individuals with typical type 1 diabetes earlier on in the pathologic process.

\section{Methods}

Participants Type 1 diabetes TrialNet, established in 2000, is an NIH-funded, international network of centres that aims to prevent type 1 diabetes and stop disease progression [10]. The TrialNet PTP is an observational study that prospectively follows non-diabetic, islet autoantibody-positive individuals (first or second-degree relatives of patients with type 1 diabetes) for the progression of islet autoimmunity and the development of clinical type 1 diabetes [11]. Out of 4041 TrialNet PTP participants enrolled between March 2004 and September 2018, we included those who, at their first (baseline) OGTT, had a $2 \mathrm{~h}$ blood glucose $\geq 11.1 \mathrm{mmol} / \mathrm{l}$ (ADA standard threshold for diabetes diagnosis if confirmed by a second test in asymptomatic individuals; see 'Procedures' below) and/or Index60 $\geq 2.00$ (threshold selected based on previous studies) $[8,9]$.

We excluded participants who had missing critical variables such as age, OGTT-stimulated C-peptide or glucose values, and islet autoantibodies or a HLA genotype. The final sample size was 354 participants, which were divided into in three mutually exclusive groups: $2 \mathrm{~h}$ glucose $\geq 11.1 \mathrm{mmol} / 1$ and Index $60<2.00[\mathrm{Glu}(+), n=76], 2 \mathrm{~h}$ glucose $<11.1 \mathrm{mmol} / 1$ and Index $60 \geq 2.00[\operatorname{Ind}(+), n=113]$, or both $2 \mathrm{~h}$ glucose $\geq 11.1 \mathrm{mmol} / 1$ and Index $60 \geq 2.00[\mathrm{Glu}(+) / \operatorname{Ind}(+), n=165]$.

All study participants provided informed consent prior to screening and enrolment, and the study was approved by the responsible ethics committee at each site.

Procedures All participants were screened for islet autoantibodies to GAD (GAD65A), insulin (microinsulin autoantibody assay [mIAA]) and insulinoma-associated antigen-2 (IA-2A). If any of these were positive at screening, autoantibodies to zinc transporter 8 (ZnT8A) and islet cell antibodies (ICA) were also tested. Once identified as being autoantibody positive, subsequent evaluations of autoantibodies for participants would include testing for all five type 1 diabetesassociated autoantibodies. Participants were monitored with autoantibody testing, $\mathrm{HbA}_{1 \mathrm{c}}$ and OGTTs at 6- or 12-month intervals depending on estimated risk [11]. Islet autoantibody [12] and C-peptide [13] assays have been previously

\section{Definitions}

Early C-peptide 30 - 0 min C-peptide during OGTT

Index60 0.3695[loge fasting C-peptide in $\mathrm{ng} / \mathrm{ml}]+0.0165[60 \mathrm{~min}$ glucose in $\mathrm{mg} / \mathrm{dl}]-0.3644[60 \mathrm{~min}$ C-peptide in $\mathrm{ng} / \mathrm{ml}]$

Overweight $\geq 85$ th percentile and $<95$ th percentile (age- and sex-adjusted from 2000 CDC growth charts)

Obese $\geq 95$ th percentile (age- and sex-adjusted from 2000 CDC growth charts)

Groups by diagnostic range OGTT at baseline (not yet diagnosed):

- $2 \mathrm{~h}$ glucose $\geq 11.1 \mathrm{mmol} / \mathrm{l}$ and Index60 $<2.00[\mathrm{Glu}(+)]$, or

- $2 \mathrm{~h}$ glucose $<11.1 \mathrm{mmol} / \mathrm{l}$ and Index60 $\geq 2.00[\operatorname{lnd}(+)]$, or

- $2 \mathrm{~h}$ glucose $\geq 11.1 \mathrm{mmol} / \mathrm{l}$ and Index60 $\geq 2.00[\mathrm{Glu}(+) / \mathrm{lnd}(+)]$

Diagnostic OGTT Within 1 month prior to or at the diagnosis of type 1 diabetes according to standard criteria 
described. HLA genotyping was performed at the Type 1 Diabetes Genetics Consortium Laboratories.

The interval of follow-up for the TrialNet PTP cohort per the study protocol is determined by the probability of being diagnosed with type 1 diabetes. OGTTs are conducted at 6-month intervals on participants with at least one of the following: $\geq 2$ positive autoantibodies, $\mathrm{HbA}_{1 \mathrm{c}} \geq 42 \mathrm{mmol} / \mathrm{mol}$, DPT-1 risk score $\geq 6.50$, fasting glucose $\geq 6.1 \mathrm{mmol} / \mathrm{lmg} / \mathrm{dl}, 30$-, 60 - or 90 -min glucose $\geq 11.1 \mathrm{mmol} / 1$, or $2 \mathrm{~h}$ glucose $\geq 7.8 \mathrm{mmol} / \mathrm{l}$. Autoantibody-positive participants who do not meet any of those criteria are followed at 12-month intervals. According to the TrialNet PTP protocol, participants with a $2 \mathrm{~h}$ glucose $\geq 11.1 \mathrm{mmol} / \mathrm{l}$ during their baseline OGTT who did not have supporting clinical criteria were to have a second ('confirmatory') OGTT to establish or rule out type 1 diabetes. Participants not meeting diagnostic criteria on the confirmatory OGTT were followed with semi-annual OGTTs. Therefore, as illustrated in the flow diagram in Fig. 1, there was a temporal discordance. $\mathrm{Glu}(+)$ and $\mathrm{Glu}(+) / \operatorname{Ind}(+)$ had confirmatory OGTTs whereas Ind(+) did not, since the latter group had not exceeded a glucose threshold that would have triggered a confirmatory OGTT in the autoantibody-positive TrialNet PTP participants.

Statistical analyses Characteristics were summarised across the overall cohort as well as by group [i.e., $\mathrm{Glu}(+)$, Ind(+) and $\mathrm{Glu}(+) / \operatorname{Ind}(+)]$ using descriptive statistics. Continuous measures were compared across all groups using Kruskal-
Wallis tests and Wilcoxon rank sum tests for pairwise comparisons between any two groups of interest. $\chi^{2}$ tests were used to evaluate differential distributions of characteristics, and factors across all three groups or between any two groups. Metabolic measures were similarly evaluated and compared between groups; differences in these measures were further assessed using graphical analyses. Generalised linear regression models were applied to further analyse differences in markers between groups, adjusting for known confounders such as age and BMI percentile. For Ind(+) participants, follow-up data from baseline were used to assess the time to type 1 diabetes diagnosis with Kaplan-Meier methodology. All analyses were performed using the statistical program R (version 3.5.1 for Windows; https://www.R-project.org/). Statistical significance was noted if two-sided $p$ values were $<0.05$.

\section{Results}

Overall, 354 participants were identified from the TrialNet PTP study who met the inclusion criteria based on their exceeding $2 \mathrm{~h}$ glucose and/or Index60 thresholds during the baseline OGTT. The median age at the baseline OGTT for the entire cohort was 11.2 years (range: $1.7-46.6$ ); $49 \%$ were male, $32 \%$ were obese or overweight, $83 \%$ were nonHispanic White, and all were relatives (first degree: 90\%) of individuals with type 1 diabetes. Participants were classified
Fig. 1 Flow diagram for TrialNet PTP participants who had diabetic range OGTTs at baseline. Out of 4041 TrialNet PTP participants enrolled between March 2004 and September 2018, we included those who, at their first (baseline) OGTT, had a $2 \mathrm{~h}$ blood glucose $\geq 11.1$ $\mathrm{mmol} / 1$ and/or Index $60 \geq 2.00$. We excluded participants who had missing critical variables such as age, OGTT-stimulated C-peptide or glucose values, and islet autoantibodies or a HLA genotype. The final sample size was 354 participants, which were divided into in three mutually exclusive groups: $2 \mathrm{~h}$ glucose $\geq 11.1 \mathrm{mmol} / 1$ and Index60 $<2.00$ [Glu(+), $n=76]$, $2 \mathrm{~h}$ glucose $<11.1 \mathrm{mmol} / \mathrm{l}$ and Index60 $\geq 2.00[\operatorname{Ind}(+), n=113]$, or both $2 \mathrm{~h}$ glucose $\geq 11.1 \mathrm{mmol} / \mathrm{l}$ and Index60 $\geq 2.00[\mathrm{Glu}(+) / \operatorname{Ind}(+)$, $n=165]$. Confirmatory OGTTs were performed in the Glu(+) and $\mathrm{Glu}(+) / \mathrm{Ind}(+)$ groups (which had a $2 \mathrm{~h}$ glucose $\geq 11.1 \mathrm{mmol} / \mathrm{l}$ at baseline) but not in the Ind(+) group

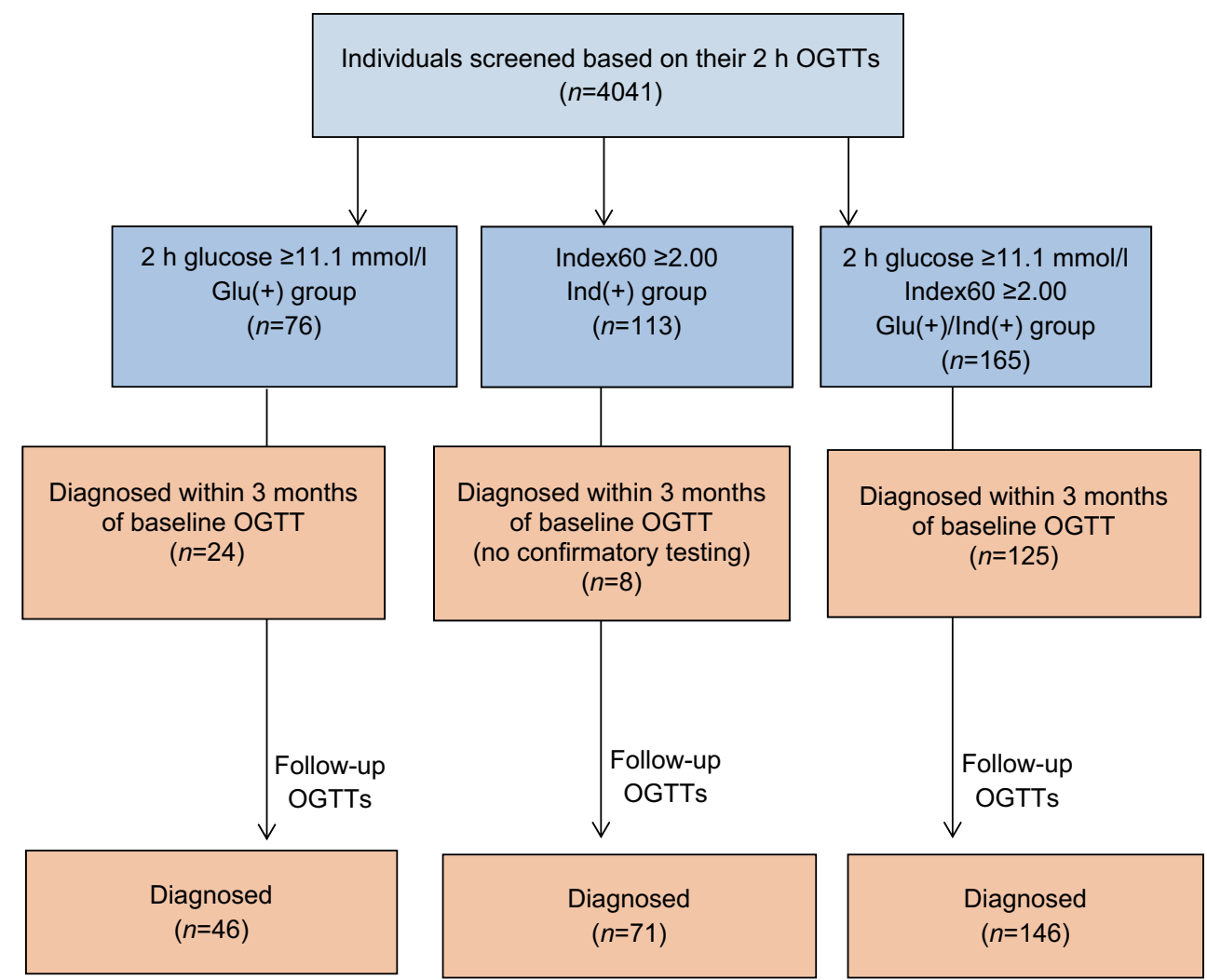


into one of three mutually exclusive groups: Glu(+), Ind(+) or $\mathrm{Glu}(+) / \mathrm{Ind}(+)$. Each section below examines differences in characteristic type 1 diabetes markers among the groups (Table 1).

Demographic/clinical Glu(+) participants were much older at the baseline OGTT than $\operatorname{Ind}(+)$ and $\mathrm{Glu}(+) / \operatorname{Ind}(+)$ participants were ( $p<0.0001$ for both comparisons). Ind $(+)$ participants had a lower mean age than $\mathrm{Glu}(+) / \mathrm{Ind}(+)$ participants did $(p=0.029)$. The prevalence of obesity or overweight in the Glu(+) group was higher than in the Ind(+) and $\mathrm{Glu}(+) / \operatorname{Ind}(+)$ groups $(p<0.0001$ for both comparisons). Participants in the Ind(+) group had a significantly lower prevalence of obesity/overweight than participants in the $\mathrm{Glu}(+) / \mathrm{Ind}(+)$ group did $(p=0.006)$.

Islet autoantibodies/HLA The Glu(+) group had a lower percentage of multiple (i.e., $\geq 2$ ) autoantibodies than either the $\operatorname{Ind}(+)$ or $\mathrm{Glu}(+) / \operatorname{Ind}(+)$ groups $\operatorname{did}(p=0.0006$ and $p<0.0001$, respectively). The frequency of mIAA positivity was lower in the $\mathrm{Glu}(+)$ group than in either the $\operatorname{Ind}(+)(p=0.003)$ or $\mathrm{Glu}(+$ )$/ \operatorname{Ind}(+)$ groups $(p=0.014)$. The $\mathrm{Glu}(+)$ group also had less ICA positivity than the $\operatorname{Ind}(+)$ group $(p=0.025)$ and less IA2 A positivity than the $\mathrm{Glu}(+) / \operatorname{Ind}(+)$ group $(p=0.015)$. We did not observe statistically significant differences in the proportions of participants carrying type 1 diabetes-associated HLA haplotypes or genotypes among the groups (Table 1).

C-peptide measures The Glu(+) group had higher values of AUC C-peptide $(p<0.0001)$, fasting C-peptide $(p<0.0001)$ and early C-peptide response $(30-0 \mathrm{~min} ; p<0.0001)$ than the Ind(+) or Glu(+)/Ind(+) groups did. The differences all remained statistically significant after adjusting for age and BMI (all $p<0.0001)$. The Ind(+) group had lower fasting $\mathrm{C}$ peptide levels $(p<0.0001)$ and greater 30-0 min C-peptide responses than the $\mathrm{Glu}(+) / \operatorname{Ind}(+)$ group $\operatorname{did}(p=0.048)$.

Figure 2 shows the consistent differences in baseline characteristics between the Glu(+) group and other groups. The $\mathrm{Glu}(+)$ group was a clear outlier among the three groups. After the exclusion of participants who had a non-first degree relative with type 1 diabetes, the differences were similar.

OGTT patterns We compared the shapes and positions of combined glucose and C-peptide response curves (GCRCs) from mean glucose and mean C-peptide values at 30, 60, 90 and $120 \mathrm{~min}$ of the baseline OGTTs among the three groups. A 2-dimensional grid with glucose on the $y$-axis and Cpeptide on the $\mathrm{X}$-axis was used to display the shapes and relative positions (Fig. 3). Visual comparisons of the groups' GCRCs revealed that the Glu(+) group had a substantially higher C-peptide response than the other two groups did; this was consistent with a significantly higher mean AUC Cpeptide compared with the other two groups (Table 1). The $\mathrm{Glu}(+) / \operatorname{Ind}(+)$ group had the most pathological OGTTs as evident from both the GCRC shape, almost monotonic indicating imminent onset, and its position on the grid, with markedly greater AUC glucose values (mean: $13.26 \mathrm{mmol} / \mathrm{l}$; SD: 2.16) than in the Glu(+) (mean: $10.82 \mathrm{mmol} / \mathrm{l}: \mathrm{SD}: 1.22$; $p<0.0001$ ) and Ind(+) (mean: $9.99 \mathrm{mmol} / \mathrm{l}$; SD: 0.83; $p<0.0001)$ groups. Interestingly, despite the difference in
Table 1 Comparisons between groups at baseline

\begin{tabular}{llll}
\hline & Glu(+) & Ind(+) & Glu(+)/Ind(+) \\
& $N=76$ & $N=113$ & $N=165$ \\
\hline Age (years) & $22.9 \pm 13.9$ & $11.8 \pm 11.0^{* * * *}$ & $14.7 \pm 10.7 * * *$ \\
Overweight or obese: $n(\%)$ & $44(58)$ & $18(16)$ & $50(30)$ \\
Missing & 0 & 2 & 1 \\
$\geq 2$ Positive islet autoantibodies: $n(\%)$ & $55(72)$ & $104(92) * *$ & $153(93)^{* * *}$ \\
mIAA positive: $n(\%)$ & $22(29)$ & $59(52)^{* *}$ & $77(47)^{*}$ \\
GADA positive: $n(\%)$ & $64(84)$ & $95(84)$ & $140(85)$ \\
IA-2A positive: $n(\%)$ & $43(57)$ & $73(65)$ & $121(73)^{*}$ \\
ICA positive: $n$ (\%) & $44(59)$ & $83(76) *$ & $112(69)$ \\
Missing & 2 & 4 & 2 \\
ZnT8A positive: $n(\%)$ & $30(54)$ & $54(65)$ & $70(59)$ \\
Not tested/missing & 20 & 30 & 46 \\
HLA DR3-DQ2/DR4-DQ8: $n(\%)$ & $13(17)$ & $31(27)$ & $41(25)$ \\
Fasting C-peptide (nmol/l) & $0.80 \pm 0.43$ & $0.33 \pm 0.17 * * *$ & $0.50 \pm 0.30^{* * *}$ \\
Early (30-0 min) C-peptide (nmol/1 min) & $1.00 \pm 0.57$ & $0.50 \pm 0.23^{* * * *}$ & $0.43 \pm 0.30^{* * *}$ \\
Mean AUC C-peptide (nmol/l) & $2.33 \pm 0.90$ & $1.13 \pm 0.37 * * *$ & $1.10 \pm 0.50^{* * *}$ \\
\hline
\end{tabular}

Values are mean (SD) unless otherwise noted

$* * * p<0.0001 ; * * p<0.01 ; * p<0.05$ in comparison to $\mathrm{Glu}(+)$ 
Fig. 2 Comparisons of characteristics among the three groups at baseline for age (a), mean AUC C-peptide (b), autoantibody positivity (c) and BMI percentile (d). Both Ind(+) and $\mathrm{Glu}(+) / \operatorname{Ind}(+)$ were significantly different $(* * * p<0.001 ; * * p<0.01$; $* p<0.05)$ from Glu(+) for all comparisons. Significant differences were present between $\operatorname{Ind}(+)$ and $\mathrm{Glu}(+) / \operatorname{Ind}(+)$ for age (a) $\left({ }^{\dagger} p<0.05\right)$ and for BMI percentile $\left({ }^{\dagger \dagger} p<0.01\right)$ a

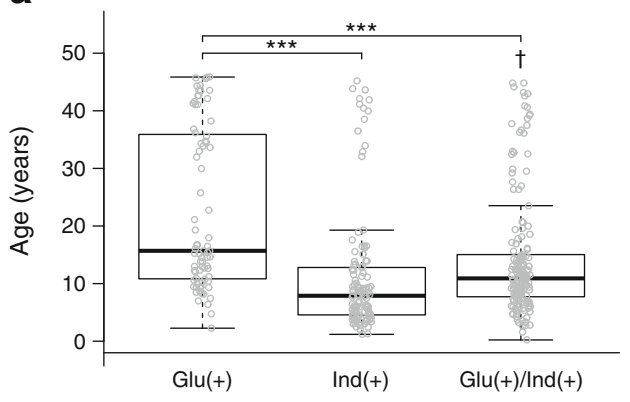

C

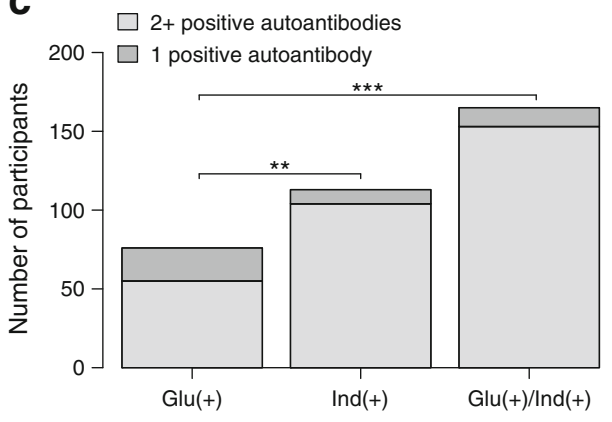

b

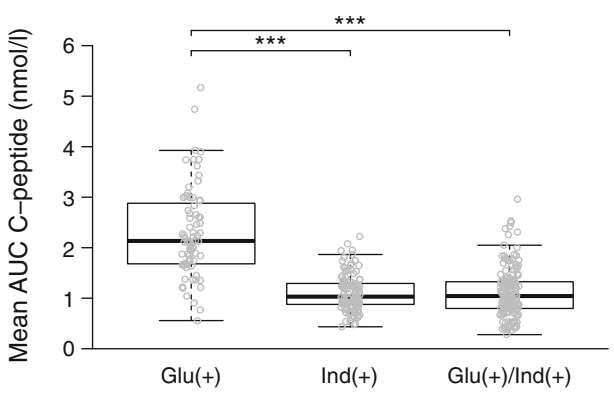

d

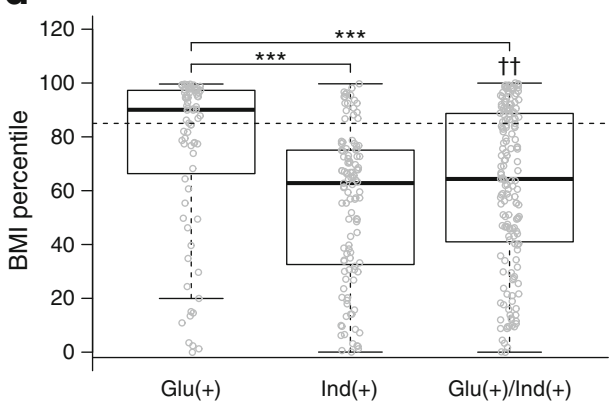

glucose values, the Ind(+) and Glu(+)/Ind(+) groups had comparable C-peptide levels (Table 1).

Figure 3 illustrates the GCRCs at baseline and at diagnosis (among those who were diagnosed by OGTT at least 3 months after baseline). It shows the evolution of shape and position of the GCRCs from baseline (dashed lines) to diagnosis (solid lines) among those with diagnostic OGTTs. Although the shape near diagnosis in the Ind $(+)$ group was much like that in the $\mathrm{Glu}(+) / \operatorname{Ind}(+)$ group, the $\mathrm{Glu}(+)$ group continued to differ substantially from the other two groups, with much higher C-peptide levels. Also, the change from baseline to diagnosis was minimal in the $\mathrm{Glu}(+) / \operatorname{Ind}(+)$ group, suggesting that diabetes was already present but not diagnosed in a number of those individuals.

Progression to type 1 diabetes The median follow-up time from baseline to the diagnosis of type 1 diabetes or end of follow-up (i.e., last OGTT) of the 354 who were studied was

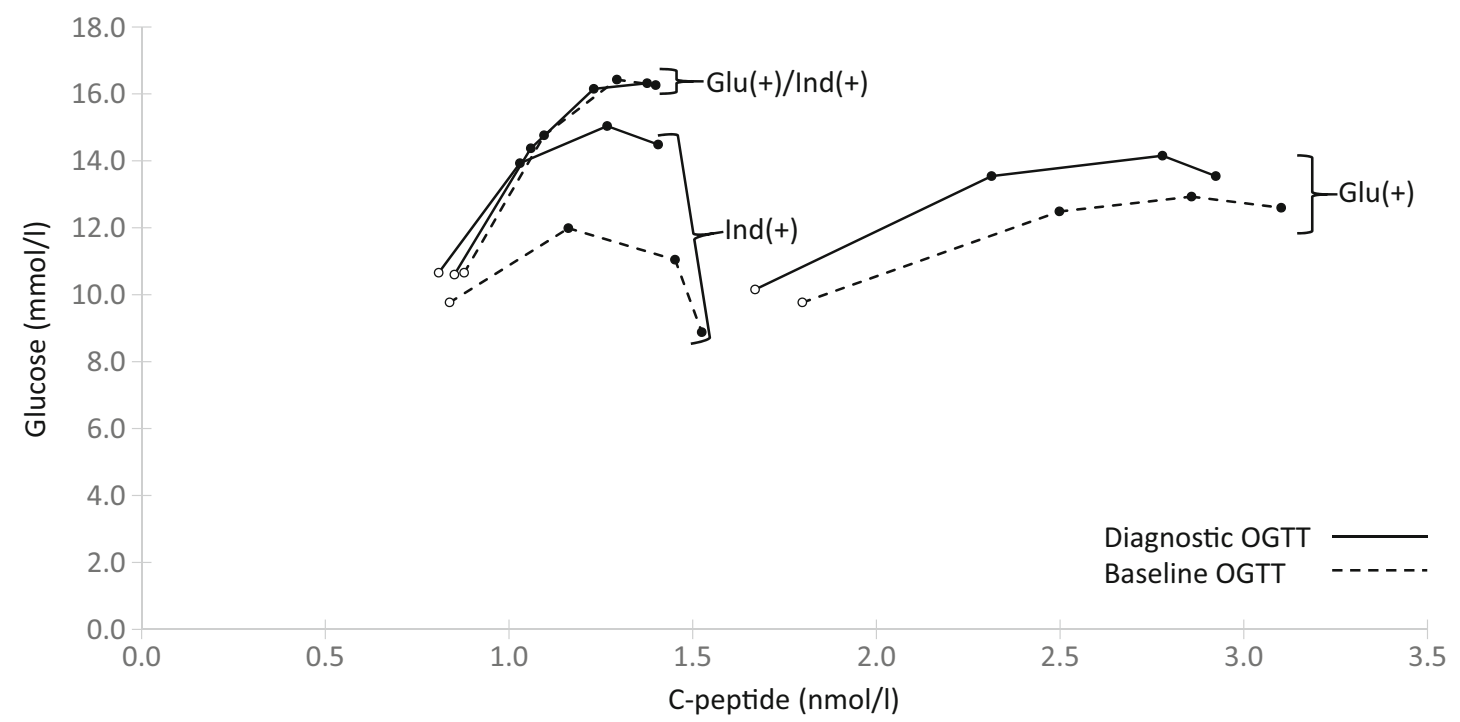

Fig. 3 Differing OGTT patterns (30 to $120 \mathrm{~min}$ ) at baseline (dashed lines) and at diagnosis of type 1 diabetes (solid lines). At baseline, C-peptide was higher at $30 \mathrm{~min}$ and had a more pronounced increase during the baseline OGTT in Glu(+) than the other two groups. At diagnosis, note the similarity between $\operatorname{Ind}(+)$ and $\mathrm{Glu}(+) / \operatorname{Ind}(+)$, and the continuing difference between Glu(+) and the other groups. Also, note that $\mathrm{Glu}(+) / \operatorname{Ind}(+)$ was essentially unchanged from baseline to the diagnosis of diabetes. Mean values of C-peptide and glucose values are shown. Glu(+)/Ind(+): $n=58, \operatorname{Ind}(+): n=39$, Glu(+): $n=34$. The empty circle at the beginning of each curve represents the values at $30 \mathrm{~min}$ in the OGTT 
3.6 years overall $(2.0,4.45$ and 2.5 years for Glu(+), Ind(+) and $\mathrm{Glu}(+) / \operatorname{Ind}(+)$, respectively). The percentage ultimately diagnosed during follow-up was $61 \%(46 / 76)$ for $\mathrm{Glu}(+)$, $63 \%(71 / 113)$ for $\operatorname{Ind}(+)$, and $88 \%(146 / 165)$ for Glu(+)/Ind(+). Ind(+) had a cumulative incidence of $95 \%$ $(95 \% \mathrm{CI} 86 \%, 98 \%)$ at 4 years. The Ind(+) cumulative incidence curve could not be compared with cumulative incidence curves from the other two groups in which confirmatory OGTTs were performed, since those with Index60 $\geq 2.00$ and a $2 \mathrm{~h}$ glucose $<11.1 \mathrm{mmol} / 1$ would not have been recognised according to the protocol for the PTP.

\section{Discussion}

In this study of autoantibody-positive individuals, we compared three mutually exclusive groups exceeding glucose and/or Index60 diagnostic thresholds for characteristics associated with type 1 diabetes. In those comparisons, we observed that participants in the $\operatorname{Ind}(+)$ and $\mathrm{Glu}(+) / \operatorname{Ind}(+)$ groups had similar characteristics, which differed substantially from those in $\mathrm{Glu}(+)$ participants and were more typical of classic type 1 diabetes (i.e., younger, less overweight/obesity, lower C-peptide measures, higher percentages of $\geq 2$ islet autoantibodies). GCRCs of Glu(+) participants also differed markedly from those of the other groups at baseline and continued to differ at diagnosis. Even though participants in the Ind(+) group did not have the same diagnostic surveillance as participants in the $\mathrm{Glu}(+)$ group, the percentages of those ultimately diagnosed were equivalent between the two groups.

During the progression from autoantibody positivity to clinical type 1 diabetes, the early C-peptide response typically decreases, which is accompanied by increasing glucose levels [14-16]. The early C-peptide response was higher in the $\mathrm{Glu}(+)$ group than in the other two groups, yet despite this seemingly greater C-peptide response, $2 \mathrm{~h}$ glucose levels were in the diabetic range. This suggests the presence of diabetogenic factors other than the autoimmune destruction of beta cells. Such factors could include insulin resistance [17, 18], adiposity, and/or other abnormalities determined by type 2 diabetes-associated genetic loci [19]. We previously demonstrated that among autoantibody-positive individuals, type 2 diabetes-associated factors, including genetic variants [20-23] and obesity $[24,25]$, can influence the progression to diabetes. Autoantibody-positive relatives carrying the type 2 diabetesassociated TCF7L2 genetic variant had lower glucose and higher C-peptide at the time of progression to diabetes [20].

It is noteworthy that when GCRCs in the Ind(+) group evolved from the baseline OGTT to the diagnostic OGTT, they had a shape (almost monotonic) and position on the 2D grid similar to the $\mathrm{Glu}(+) / \operatorname{Ind}(+)$ group, which changed minimally (Fig. 3). This observation suggests that, at the baseline OGTT, participants in the $\mathrm{Glu}(+) / \mathrm{Ind}(+)$ group had more advanced progression to type 1 diabetes than participants in the $\operatorname{Ind}(+)$ group did. It is possible that most $\mathrm{Glu}(+) / \operatorname{Ind}(+)$ participants who had confirmatory OGTTs could already have reached the onset of disease, which would have been unrecognised without Index60 as a criterion. Thus, it appears that the two groups shared the same pathway towards type 1 diabetes on the 2D grid: GCRCs in the Ind(+) group resembled those in the Glu(+)/Ind(+) group when there was further decompensation and glucose levels increased substantially. In contrast, GCRCs in the Glu(+) group differed markedly from the other two groups at baseline and diagnosis, which suggests that the differences between the Glu(+) group and the other groups were not a function of disease progression but rather pathogenesis. The differing GCRCs in the Glu(+) group are consistent with the atypical associations with markers of type 1 diabetes in that group (e.g., age, adiposity, autoantibody positivity).

A limitation of this study was the use of HLA DR3-DQ2 and DR4-DQ8 alone to evaluate genetic differences. Although we observed a greater frequency of these high-risk haplotypes in the $\operatorname{Ind}(+)$ and $\mathrm{Glu}(+) / \operatorname{Ind}(+)$ groups than in the $\mathrm{Glu}(+)$ group, the differences did not reach statistical significance. Type 1 diabetes genetic risk scores could have been helpful in showing differences, since they have demonstrated superiority over HLA alone to predict type 1 diabetes in at-risk individuals [26]. However, the number of participants characterised with a genetic risk score was insufficient for analysis. Individual alleles at other loci have smaller effects on risk and therefore would be unlikely to show significant associations in our analysis. Another limitation was the inability to assess insulin resistance, since measures such as HOMA-IR have not been validated in individuals with severe insulin deficiency, as well as in other populations. Bias must always be considered in observational studies. In our study, bias could have been introduced by the temporal discordance resulting from the lack of confirmatory testing of Index60 values $\geq 2.00$. However, such bias would likely have served against Index60, since Ind(+) had less diagnostic surveillance.

The contrasts between the $\mathrm{Glu}(+)$ and $\mathrm{Ind}(+)$ groups are revealing and suggest a need to reassess the current diagnostic criteria for type 1 diabetes. Glu(+), atypical for characteristics of type 1 diabetes, represented $32 \%$ (76/241) of those with a $2 \mathrm{~h}$ glucose $\geq 11.1 \mathrm{mmol} / \mathrm{l}$ at the baseline OGTT. If Ind(+) had also been included as a diagnostic criterion for the baseline OGTTs in the PTP, an additional 47\% (354/241) of participants would have warranted confirmatory testing for diagnosis. Those additional Ind(+) individuals would have been more typical for type 1 diabetes with as much likelihood for acquiring the disorder as Glu(+) individuals were. Thus, it appears that $\operatorname{Ind}(+)$, or a comparable glucose and C-peptide composite measure, would be as justified as a diagnostic criterion for type 1 diabetes as $\mathrm{Glu}(+)$. 
The addition of $\operatorname{Ind}(+)$ as a diagnostic criterion would provide several benefits. The greater typicality of Ind $(+)$ could enhance the performance of potential immunomodulatory therapies. For example, as a diagnostic criterion, Ind(+) would provide a younger study population, which in itself is a predictor of response to most immunomodulatory therapies in autoantibody-positive relatives [6]. More individuals would be diagnosed earlier, which could enhance studies designed to prevent or delay the decline of insulin secretion. Importantly, an earlier diagnosis could potentially decrease the frequency of diabetic ketoacidosis. Also, the addition of $\operatorname{Ind}(+)$ as a diagnostic criterion could provide a potential means for classifying metabolic heterogeneity at diagnosis and help to personalise clinical management.

The findings from this study raise the important question of whether $\mathrm{Glu}(+)$ is characteristic of the type 1 diabetes spectrum or whether it represents a different disorder such as type 2 diabetes. We have previously demonstrated that type 2 diabetesassociated mechanisms, such as obesity and genetic factors, can influence progression of islet autoimmunity [21,27] and development of clinical diabetes $[21,24,25]$ in autoantibodypositive individuals. It is plausible that some of those mechanisms are at play in the subset of $\mathrm{Glu}(+)$ participants. Other questions also arise. How do individuals in this group relate to latent autoimmune diabetes in adults (LADA) [28]? Should Glu(+) be differentiated from Ind(+) with regard to glucose management? Should Glu(+) be considered separately in designing protocols for preventing further insulin secretory decline after diagnosis? Also, should Index60 be incorporated among the criteria for staging of type 1 diabetes? Regarding the last question, the findings from our prior studies and the current study appear to suggest that a composite glucose/C-peptide measure such as Index60, would enhance the staging of type 1 diabetes. Future studies, including longitudinal observations after the clinical diagnosis, will need to address these questions.

In conclusion, the findings of this study appear to indicate that autoantibody-positive individuals who exceed the $2 \mathrm{~h}$ glucose $11.1 \mathrm{mmol} / 1$ threshold for diabetes, but not the $\geq 2.00$ Index60 threshold, have distinct GCRCs from those exceeding Index60 $\geq 2.00$ with or without a $2 \mathrm{~h}$ glucose $\geq 11.1 \mathrm{mmol} / 1$. Individuals reaching the $\geq 2.00$ Index 60 threshold with a $2 \mathrm{~h}$ glucose still $<11.1 \mathrm{mmol} / \mathrm{l}$ appear to already be at or near the stage of clinical type 1 diabetes (stage 3) [29], warranting confirmatory testing for type 1 diabetes, but with less beta cell impairment compared with those who also have glucose criteria. These findings provide evidence that diagnostic criteria for type 1 diabetes should include Index60 or a similar composite measure of glucose and C-peptide.

Supplementary Information The online version contains peer-reviewed but unedited supplementary material available at https://doi.org/10.1007/s00125020-05365-4.
Acknowledgements Parts of the content of this manuscript were communicated as an oral presentation at the ADA 79th Scientific Meeting in San Francisco, CA, in June 2019.

Data availability TrialNet data can be requested from the NIDDK public repository. The datasets generated and analysed during the current study will be made available by request from the NIDDK Central Repository at https://repository.niddk.nih.gov/studies/trialnet

Funding The sponsor of the trial was the Type 1 diabetes TrialNet Study Group. Type 1 diabetes TrialNet Study Group is a clinical trials network funded by the National Institutes of Health (NIH) through the National Institute of Diabetes and Digestive and Kidney Diseases, the National Institute of Allergy and Infectious Diseases, and The Eunice Kennedy Shriver National Institute of Child Health and Human Development, through the cooperative agreements U01 DK061010, U01 DK061034, U01 DK061042, U01 DK061058, U01 DK085465, U01 DK085453, U01 DK085461, U01 DK085466, U01 DK085499, U01 DK085504, U01 DK085509, U01 DK103180, U01 DK103153, U01 DK085476, U01 DK103266, U01 DK103282, U01 DK106984, U01 DK106994, U01 DK107013, U01 DK107014, UC4 DK106993 and the JDRF. The contents of this article are solely the responsibility of the authors and do not necessarily represent the official views of the NIH or the JDRF.

Authors' relationships and activities The authors declare that there are no relationships or activities that might bias, or be perceived to bias, their work.

All authors are members of the Type 1 diabetes TrialNet Study Group (list of study group members in provided as electronic supplementary material [ESM]). MJR and JS are the guarantors of this article and take full responsibility for the work as a whole, including the study design, access to data and the decision to submit and publish the manuscript.

Contribution statement MJR contributed to study design as well as data analysis and interpretation, and wrote the first draft of the manuscript. BMN, LMJ, ES, AP, DAS, MAA, JS and JP contributed to data interpretation and revised the manuscript. SG and LEB conducted data analysis and contributed to data interpretation and manuscript revisions. JMS conceptualised and designed the study, and contributed to data analysis and interpretation, and manuscript revisions. All authors have approved the version of the manuscript to be published.

\section{References}

1. American Diabetes Association (2020) 2. Classification and Diagnosis of Diabetes. Diabetes Care 43(Suppl 1):S14-S31. https://doi.org/10.2337/dc20-S002

2. Noble JA, Valdes AM, Varney MD et al (2010) HLA class I and genetic susceptibility to type 1 diabetes: results from the Type 1 Diabetes Genetics Consortium. Diabetes 59(11):2972-2979. https://doi.org/10.2337/db10-0699

3. Katsarou A, Gudbjörnsdottir S, Rawshani A et al (2017) Type 1 diabetes mellitus. Nat Rev Dis Primers 3:17016. https://doi.org/10. 1038/nrdp.2017.16

4. Greenbaum CJ, Beam CA, Boulware D et al (2012) Fall in Cpeptide during first 2 years from diagnosis: evidence of at least two distinct phases from composite Type 1 Diabetes TrialNet data. Diabetes 61(8):2066-2073. https://doi.org/10.2337/db11-1538

5. Davis AK, DuBose SN, Haller MJ et al (2015) Prevalence of detectable C-Peptide according to age at diagnosis and duration of type 1 diabetes. Diabetes Care 38(3):476-481. https://doi.org/10.2337/ dc14-1952 
6. Leete P, Mallone R, Richardson SJ, Sosenko JM, Redondo MJ, Evans-Molina C (2018) The effect of age on the progression and severity of type 1 diabetes: potential effects on disease mechanisms. Curr Diab Rep 18(11):115. https://doi.org/10.1007/s11892-0181083-4

7. Battaglia M, Ahmed S, Anderson MS et al (2020) Introducing the endotype concept to address the challenge of disease heterogeneity in type 1 diabetes. Diabetes Care 43(1):5-12. https://doi.org/10. 2337/dc19-0880

8. Sosenko JM, Skyler JS, DiMeglio LA et al (2015) A new approach for diagnosing type 1 diabetes in autoantibody-positive individuals based on prediction and natural history. Diabetes Care 38(2):271276. https://doi.org/10.2337/dc14-1813

9. Nathan BM, Boulware D, Geyer S et al (2017) Dysglycemia and index60 as prediagnostic end points for type 1 diabetes prevention trials. Diabetes Care 40(11):1494-1499. https://doi.org/10.2337/ de17-0916

10. Skyler JS, Greenbaum CJ, Lachin JM et al (2008) Type 1 diabetes TrialNet-an international collaborative clinical trials network. Ann N Y Acad Sci 1150:14-24. https://doi.org/10.1196/annals.1447. 054

11. Mahon JL, Sosenko JM, Rafkin-Mervis L et al (2009) The TrialNet Natural History Study of the Development of Type 1 Diabetes: objectives, design, and initial results. Pediatr Diabetes 10(2):97104. https://doi.org/10.1111/j.1399-5448.2008.00464.x

12. Fouts A, Pyle L, Yu L et al (2016) Do electrochemiluminescence assays improve prediction of time to type 1 diabetes in autoantibody-positive TrialNet subjects? Diabetes Care 39(10): 1738-1744. https://doi.org/10.2337/dc16-0302

13. Little RR, Rohlfing CL, Tennill AL et al (2008) Standardization of C-peptide measurements. Clin Chem 54(6):1023-1026. https://doi. org/10.1373/clinchem.2007.101287

14. Sosenko JM, Palmer JP, Rafkin LE et al (2010) Trends of earlier and later responses of C-peptide to oral glucose challenges with progression to type 1 diabetes in diabetes prevention trial-type 1 participants. Diabetes Care 33(3):620-625. https://doi.org/10.2337/ dc09-1770

15. Sosenko JM, Skyler JS, Krischer JP et al (2010) Glucose excursions between states of glycemia with progression to type 1 diabetes in the diabetes prevention trial-type 1 (DPT-1). Diabetes 59(10): 2386-2389. https://doi.org/10.2337/db10-0534

16. Ismail HM, Xu P, Libman IM et al (2018) The shape of the glucose concentration curve during an oral glucose tolerance test predicts risk for type 1 diabetes. Diabetologia 61(1):84-92. https://doi.org/ 10.1007/s00125-017-4453-6

17. Fourlanos S, Narendran P, Byrnes GB, Colman PG, Harrison LC (2004) Insulin resistance is a risk factor for progression to type 1 diabetes. Diabetologia 47(10):1661-1667. https://doi.org/10.1007/ s00125-004-1507-3

18. Bingley PJ, Mahon JL, Gale EA, Group ENDIT (2008) Insulin resistance and progression to type 1 diabetes in the European
Nicotinamide Diabetes Intervention Trial (ENDIT). Diabetes Care 31(1):146-150. https://doi.org/10.2337/dc07-0103

19. Redondo MJ, Evans-Molina C, Steck AK, Atkinson MA, Sosenko $J$ (2019) The influence of type 2 diabetes-associated factors on type 1 diabetes. Diabetes Care 42(8):1357-1364. https://doi.org/10. 2337/dc19-0102

20. Redondo MJ, Geyer S, Steck AK et al (2018) Genetic variants contribute to phenotypic heterogeneity of type 1 diabetes. Diabetes Care 41(2):311-317. https://doi.org/10.2337/dc17-0961

21. Redondo MJ, Steck AK, Sosenko J et al (2018) Transcription factor 7-like 2 (TCF7L2) gene polymorphism and progression from single to multiple autoantibody positivity in individuals at risk for type 1 diabetes. Diabetes Care 41(12):2480-2486. https://doi.org/10. 2337/dc18-0861

22. Redondo MJ, Grant SF, Davis A, Greenbaum C, Biobank TDE (2017) Dissecting heterogeneity in paediatric Type 1 diabetes: association of TCF7L2 rs7903146 TT and low-risk human leukocyte antigen (HLA) genotypes. Diabet Med 34(2):286-290. https://doi. org/10.1111/dme.13123

23. Redondo MJ, Muniz J, Rodriguez LM et al (2014) Association of TCF7L2 variation with single islet autoantibody expression in children with type 1 diabetes. BMJ Open Diabetes Res Care 2(1): e000008. https://doi.org/10.1136/bmjdrc-2013-000008

24. Ferrara CT, Geyer SM, Evans-Molina C et al (2017) The role of age and excess body mass index in progression to type 1 diabetes in atrisk adults. J Clin Endocrinol Metab 102(12):4596-4603. https:// doi.org/10.1210/jc.2017-01490

25. Ferrara CT, Geyer SM, Liu YF et al (2017) Excess BMI in childhood: a modifiable risk factor for type 1 diabetes development? Diabetes Care 40(5):698-701. https://doi.org/10.2337/dc16-2331

26. Redondo MJ, Geyer S, Steck AK et al (2018) A type 1 diabetes genetic risk score predicts progression of islet autoimmunity and development of type 1 diabetes in individuals at risk. Diabetes Care 41(9):1887-1894. https://doi.org/10.2337/dc18-0087

27. Ferrara-Cook C, Geyer SM, Evans-Molina C et al (2020) Excess BMI accelerates islet autoimmunity in older children and adolescents. Diabetes Care 43(3):580-587. https://doi.org/10.2337/dc191167

28. Mishra R, Hodge KM, Cousminer DL, Leslie RD, Grant SFA (2018) A global perspective of latent autoimmune diabetes in adults. Trends Endocrinol Metab 29(9):638-650. https://doi.org/ 10.1016/j.tem.2018.07.001

29. Insel RA, Dunne JL, Atkinson MA et al (2015) Staging presymptomatic type 1 diabetes: a scientific statement of JDRF, the Endocrine Society, and the American Diabetes Association. Diabetes Care 38(10):1964-1974. https://doi.org/10.2337/dc151419

Publisher's note Springer Nature remains neutral with regard to jurisdictional claims in published maps and institutional affiliations. 